\title{
Effect of Add-On Therapy of Sodium-Glucose Cotransporter 2 Inhibitors and Dipeptidyl Peptidase 4 Inhibitors on Adipokines in Type 2 Diabetes Mellitus
}

\author{
Abid Shaheer $^{\mathrm{a}, \mathrm{e}} \mathbb{( \mathbb { D }}$, Ashok Kumar $^{\mathrm{b}}$, Palat Menon ${ }^{\mathrm{c}}$, Mahir Jallo ${ }^{\mathrm{d}}$, Shaikh Basha ${ }^{\mathrm{d}}$
}

\begin{abstract}
Background: Excess adiposity is associated with an increased risk of cardiovascular disease due to metabolic changes in the body. Visceral obesity increases the risk of diabetes mellitus through adipocytokines and hence the effective targeting therapies are essential to control obesity in high-risk individuals. The study's main objective was to evaluate the effect of add-on therapy of sodium-glucose cotransporter 2 (SGLT2) inhibitors and dipeptidyl peptidase 4 (DPP4) inhibitors on visceral fat-associated serum adipokines.
\end{abstract}

Methods: The study included 90 subjects diagnosed with type 2 diabetes mellitus. The blood samples were taken before starting first-line therapy with metformin, 12 weeks after starting metformin therapy and 12 weeks after starting add-on therapy. Serum adipokines were analyzed with enzyme-linked immunosorbent assay (ELISA). Hemoglobin A1c (HbA1c) level was estimated with high-performance liquid chromatography (HPLC). The biochemical variables were measured using Cobas ${ }^{\circledR} 6000$ analyzer.

Results: The mean adiponectin level was significantly elevated with add-on therapy using SGLT2 inhibitors and DPP4 inhibitors $(\mathrm{P}<$ $0.001)$. The mean retinol binding protein 4 (RBP4), fatty acid binding protein 4 (FABP4) and visfatin levels were reduced considerably ( $\mathrm{P}$ $<0.001)$. The SGLT2 inhibitors are more effective on serum FABP4 in patients with type 2 diabetes $(\mathrm{P}=0.038)$. The mean fasting plasma glucose (FPG), postprandial blood glucose (PPBG) and HbA1c levels were reduced significantly with add-on therapy $(\mathrm{P}<0.001)$. Lipid pro-

Manuscript submitted April 20, 2021, accepted May 7, 2021

Published online June 25, 2021

aDepartment of Biomedical Sciences, Gulf Medical University, Ajman, United Arab Emirates

bDepartment of Biochemistry, Rajah Muthiah Medical College, Annamalai University, Chidambaram, India

${ }^{c}$ Clinical Department, Pathology Laboratory, Fakeeh University Hospital, Dubai, United Arab Emirates

dDepartment of Internal Medicine, Gulf Medical University, Ajman, United Arab Emirates

${ }^{e}$ Corresponding Author: Abid Shaheer, Department of Biomedical Sciences, Gulf Medical University, PO Box 4184, Ajman, United Arab Emirates.

Email: abidshaheer@gmail.com

doi: https://doi.org/10.14740/jocmr4510 file was also altered significantly with this add-on therapy $(\mathrm{P}<0.001)$.

Conclusions: The results indicate that add-on therapy exerts a beneficial effect in type 2 diabetic patients insufficiently controlled with metformin only by altering the visceral fat-associated adipokine levels and controlling the metabolic activities.

Keywords: Adipokines; Visceral fat; Metformin; SGLT2 inhibitors; DPP4 inhibitors

\section{Introduction}

Type 2 diabetes mellitus is a metabolic disorder that occurs primarily due to the impaired insulin production from the pancreatic $\beta$ cells and peripheral insulin resistance [1]. In visceral obesity, the excessive intra-abdominal fat impairs health. It is a specific independent risk factor associated strongly with the pathogenesis of insulin resistance, leading to type 2 diabetes mellitus [2]. Excess adiposity is associated with an increased risk of cardiovascular disease due to blood pressure changes, alteration in lipid metabolism and uncontrolled blood glucose $[3,4]$. Visceral obesity increases the risk of diabetes mellitus through several adipocytokines and hence the effective targeting therapies are essential to control obesity in high-risk individuals [5]. Adipokines are a group of bioactive cytokines primarily secreted by adipose tissue. The imbalance in adipokines production leads to the pathogenesis of obesity-linked metabolic disorders and their complications [6].

The recent evaluation conducted by the International Diabetes Federation (IDF) revealed the number of adult populations affected by diabetes mellitus in the Middle East was 54.8 million, predicted to increase 76 million by 2030 [7]. The incidence of obesity, type 2 diabetes, hypertension and dyslipidemia is a significant health problem in the United Arab Emirates [8].

Metformin is a first-line oral hypoglycemic drug that reduces glucose production in the liver, decreases the intestinal absorption of glucose and improves insulin sensitivity by up-regulation of glucose transporters that promotes glucose uptake and utilization $[9,10]$. Metformin activates adenosine monophosphate-activated protein kinase by the upstream liver kinase B1 or increasing adenosine monophosphate/adenosine triphosphate ratio by inhibiting mitochondrial respiration. Metformin also acts on glycerol 
metabolism by blocking mitochondrial glycerophosphate dehydrogenase. Metformin alters the intestinal microbes in humans, but its significance in glucose metabolism is still unclear [9-11].

The sodium-glucose cotransporter 2 (SGLT2) is a transporter located in the proximal renal tubule, which helps to reabsorb $90 \%$ of the glucose filtered by the capillaries of the glomerulus. The SGLT2 inhibitors are a group of medication that plays a vital role in decreasing renal glucose reabsorption by blocking the action of SGLT2, thereby increasing urinary glucose excretion. These drugs have received approval in the treatment of type 2 diabetes mellitus [12]. SGLT2 inhibitors are the most beneficial antihyperglycemic medication currently used as an add-on therapy with metformin in patients with a history of cardiovascular or renal disease to control their blood glucose level [13].

SGLT2 inhibitors are the best choice of therapy for obese patients. These drugs help to reduce body fat and have an influential role in controlling cardiovascular risk in type 2 diabetes mellitus [14]. Dapagliflozin reduces the secretion of proinflammatory chemokines effectively and improves epicardial adipose tissue cells' differentiation in patients with cardiovascular disease [15]. Add-on therapy of dapagliflozin and empagliflozin with metformin is safe, effective and well-tolerated. The side effects of these combination therapies are infrequent when compared with monotherapy $[16,17]$.

Dipeptidyl peptidase 4 (DPP4) is a multifunctional adipocytokine mainly released by fully differentiated adipocytes. DPP4 is a marker for metabolic syndrome, visceral obesity and insulin resistance [18]. DPP4 inhibitors are currently used for glycemic control and preserve $\beta$-cell function in type 2 diabetic patients. DPP4 inhibitors enhance glucagon-like peptide- 1 and glucose-dependent insulin-tropic polypeptide which leads to increased insulin secretion by $\beta$ cells of the pancreas and reduced glucagon secretion which in turn lowers glucose production in the liver [19]. Add-on therapy of sitagliptin and vildagliptin is also safe and well-tolerated when combined with metformin [20-22].

The present study primarily focuses on the effect of addon therapy of SGLT2 inhibitors and DPP4 inhibitors on visceral fat-associated adipokines.

\section{Materials and Methods}

\section{Study population}

The study subjects include males and females in the age group 30 - 60 years, diagnosed with type 2 diabetes mellitus in Internal Medicine Department, Thumbay Hospital, Ajman, United Arab Emirates. The study focussed on type 2 diabetic patients who are on metformin monotherapy. The study excluded type 1 diabetes mellitus, gestational diabetes mellitus, diabetic ketoacidosis, moderate to severe renal disease and liver disease.

\section{Sample size}

A total of 90 adults with type 2 diabetes mellitus were included in the study.

\section{Study settings}

Type 2 diabetic patients were identified according to the American Diabetic Association (ADA) diagnostic criteria. The data were taken at baseline and the subjects were observed for a minimum of 12 weeks to confirm whether they are under control on metformin monotherapy. The glycemic control was monitored by estimating fasting plasma glucose (FPG), 2-h postprandial blood glucose (PPBG) and hemoglobin A1c (HbA1c) levels. The study subjects who achieved HbAlc < $7 \%$ or $1 \%$ reduction in $\mathrm{HbA} 1 \mathrm{c}$ level were excluded. The data were collected again after 12 weeks of first-line therapy and after 12 weeks of add-on therapy of SGLT2 inhibitors or DPP4 inhibitors with metformin. A questionnaire was used to record participants' details including lifestyle parameters and family history, after obtaining written informed consent. The blood pressure, waist circumference (WC), height, weight and body mass index (BMI) were recorded.

\section{Duration of study}

The duration of the study was 2 years after approval from the Institutional Ethics Committee (IRB/COM/FAC/05/2018). The research work was done from April 2018 to March 2020.

\section{Study instrument and validation procedure}

The ethical approval was taken from our the Institutional Ethics Committee. Informed written consent was obtained from all individual participants and the confidentiality of study subjects, including their details were maintained. This study was conducted in compliance with the ethical standards of the Gulf Medical University, Ajman, United Arab Emirates.

\section{Methodology}

Venous blood samples collected from each of the study subjects were analyzed for FPG, 2-h PPBG, total cholesterol (TC), LDL cholesterol (LDL-C), HDL cholesterol (HDL-C), triglycerides (TG), creatinine, albumin, aspartate transaminase (AST) and alanine transaminase (ALT) on Cobas ${ }^{\circledR} 6000$ analyzer (Roche). High-performance liquid chromatography (HPLC) was used to estimate HbA1c level.

Serum adipokines including retinol binding protein 4 (RBP4), fatty acid binding protein 4 (FABP4), visfatin and adiponectin were analyzed by enzyme-linked immunosorbent assay (ELISA) with the reagents supplied by BioVendor, Laboratory medicina a.s, Czech Republic. BMI was calculated by dividing the weight (in $\mathrm{kg}$ ) by height ${ }^{2}$ (in $\mathrm{m}^{2}$ ). A sphygmomanometer was used to measure systolic and diastolic blood pressure.

\section{Data analysis}

The data were expressed as the mean \pm standard deviation or 
Table 1. Baseline Characteristics, Family History and Clinical Variables Between the Study Subjects

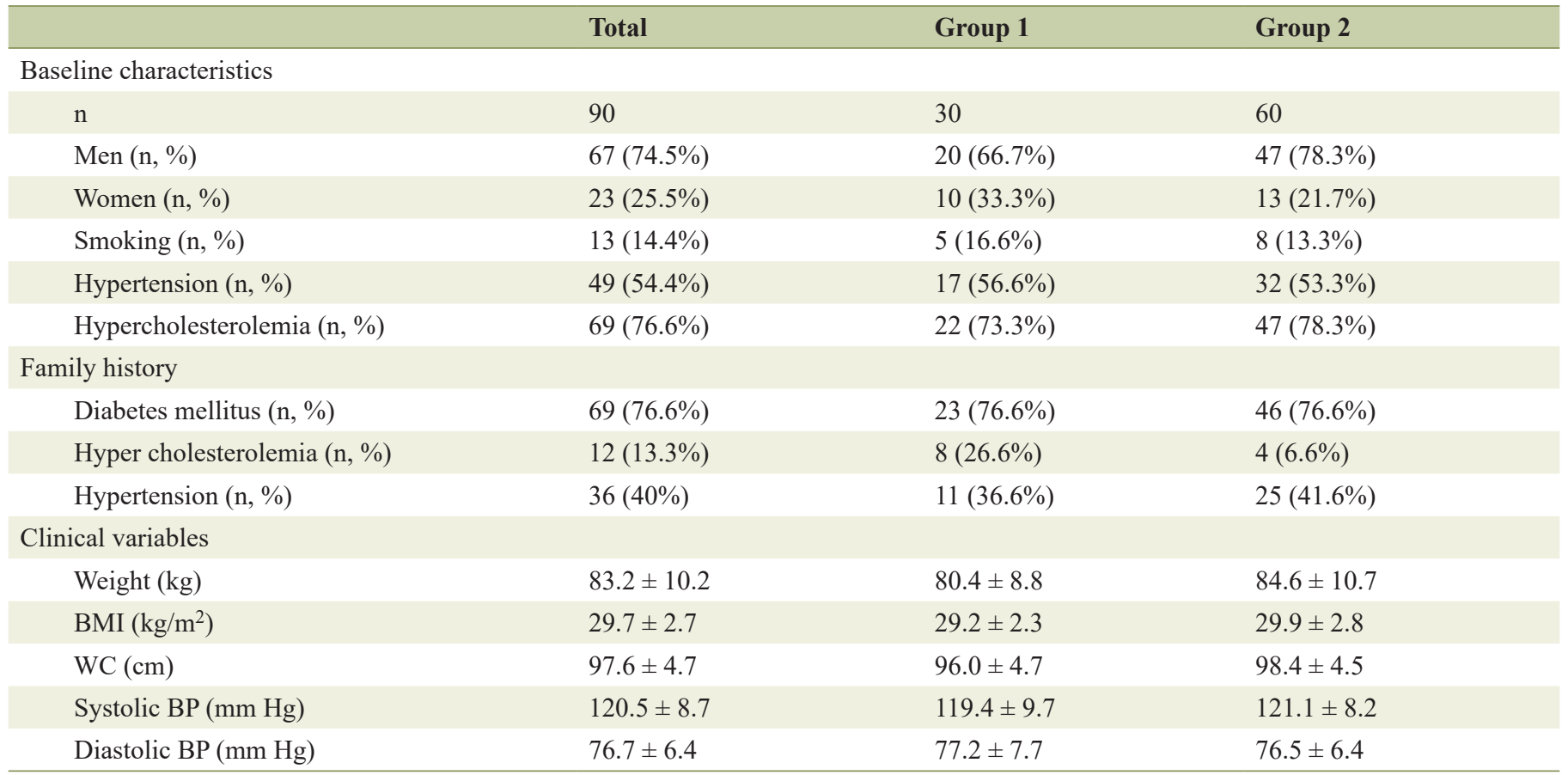

Data are expressed as means \pm SD, $n$ (\%). Group 1: metformin + DPP4 inhibitors. Group 2: metformin + SGLT2 inhibitors. DPP4: dipeptidyl peptidase 4; SGLT2: sodium-glucose cotransporter 2; BMI: body mass index; WC: waist circumference; BP: blood pressure.

frequencies. Parametric and non-parametric tests were applied to compare groups according to the type of variable and normality distribution. The z-test was applied to evaluate the differences between the means across the groups. The MannWhitney $U$ test was used for non-normally distributed variables. Repeated measures analysis of covariance (ANCOVA) was applied to find the effect of SGLT2 inhibitor and DPP4 inhibitor on the clinical parameters, biochemical variables and adipokines. Data were analyzed using the Statistical Package for Social Science (SPSS Version 27.0) and a P-value $<0.05$ was considered significant.

\section{Results}

Out of the 90 participants, $67(74.5 \%)$ were male and 23 $(25.5 \%)$ were female. Table 1 outlines the baseline characteristics, family history and clinical variables between the study subjects. The mean age of participants was $46.0 \pm 7.7$ years, the mean weight was $83.2 \pm 10.2 \mathrm{~kg}$, mean BMI was $29.7 \pm$ $2.7 \mathrm{~kg} / \mathrm{m}^{2}$ and mean WC was $97.6 \pm 4.7 \mathrm{~cm}$. The mean systolic and diastolic blood pressures were $120.5 \pm 8.7$ and $76.7 \pm 6.4$ $\mathrm{mm} \mathrm{Hg}$, respectively. Out of the 90 participants, $14.5 \%$ were smokers and $85.5 \%$ were non-smokers. It is also shown that $54.5 \%$ had a history of hypertension and $76.5 \%$ had hypercholesterolemia. The participants had an inactive lifestyle and were overweight or obese in all the study groups. Out of the total 90 participants, $77 \%$ had a family history of diabetes mellitus, $13.3 \%$ had a family history of hypercholesterolemia and $40 \%$ had a family history of hypertension.
Table 2 outlines the groups and treatment modality of the study participants. All the study subjects were taken metformin $1,000 \mathrm{mg}$ (Glucophage ${ }^{\circledR}$ ) twice/day as first-line treatment for 12 months, followed by add-on therapy for 12 weeks. Among the participants, 30 subjects had taken DPP4 inhibitors, and 60 subjects had taken SGLT2 inhibitors. The 30 subjects who received add-on therapy of DPP4 inhibitors included 15 participants with sitagliptin/metformin $50 \mathrm{mg} / 1,000 \mathrm{mg}$ (Janumet $^{\circledR}$ ) and 15 with vildagliptin/metformin $50 \mathrm{mg} / 1,000 \mathrm{mg}$ (Galvusmet $^{\mathbb{R}}$ ). The 60 subjects who received add-on therapy of SGLT2 inhibitors included 15 participants with dapagliflozin/ metformin $5 \mathrm{mg} / 1,000 \mathrm{mg}$ (Xigduo ${ }^{\circledR}$ ), 15 with dapagliflozin/ metformin $10 \mathrm{mg} / 1,000 \mathrm{mg}$ (Xigduo ${ }^{\circledR}$ ), 15 with empagliflozin/ metformin $5 \mathrm{mg} / 1,000 \mathrm{mg}$ (Synjardy ${ }^{\circledR}$ ) and 15 with empagliflozin/metformin $12.5 \mathrm{mg} / 1,000 \mathrm{mg}\left(\right.$ Synjardy $\left.{ }^{\circledR}\right)$.

Supplementary Material 1 (wwww.jocmr.org) shows the effect of add-on therapy of DPP4 inhibitors and SGLT2 inhibitors on clinical parameters, biochemical variables and adipokines. Add-on therapy significantly reduced the mean weight by $-1.60 \%$ (95\% confidence interval $(\mathrm{CI}):-1.85,-1.35)$, $1.29 \%(-1.83,-0.76)$ and $-1.91 \%(95 \%$ CI: $-2.16,-1.67)$ when compared to metformin monotherapy $(\mathrm{P}<0.001)$ within the total study subjects, groups 1 and 2 respectively. Add-on therapy significantly altered the mean BMI by -0.54 (95\% CI: -0.65 , -0.43 ), -0.40 (95\% CI: $-0.68,-0.12$ ) and -0.68 (95\% CI: -0.77 , $-0.59)$ from 12 th week $(\mathrm{P}<0.05)$ within the total study subjects, groups 1 and 2 respectively. Add-on therapy also exerted a beneficial change in the mean WC by -2.49 (95\% CI: -2.67 , $-2.31),-2.11$ (95\% CI: $-2.41,-1.81)$ and -2.87 (95\% CI: -3.08 , -2.66) when compared to metformin monotherapy $(\mathrm{P}<0.001)$ 
Table 2. The Groups and Treatment Modality of the Study Participants

\begin{tabular}{|c|c|c|c|c|}
\hline Group & Number of subjects & Firstline therapy & Add-on therapy & Daily dosage \\
\hline \multirow[t]{2}{*}{$\begin{array}{l}1 \text { (metformin }+ \\
\text { DPP4 inhibitors) }\end{array}$} & 30 & $\begin{array}{l}\text { Metformin 1,000 } \\
\left.\text { mg (Glucophage }{ }^{\circledR}\right)\end{array}$ & $\begin{array}{l}\text { Sitagliptin/metformin } 50 \\
\mathrm{mg} / 1,000 \mathrm{mg}\left(\text { Janumet }^{\mathbb{R}}\right)\end{array}$ & One tablet two times per day \\
\hline & & & $\begin{array}{l}\text { Vildagliptin/metformin } 50 \\
\mathrm{mg} / 1,000 \mathrm{mg}\left(\text { Galvusmet }^{\circledR}\right)\end{array}$ & One tablet two times per day \\
\hline \multirow[t]{3}{*}{$\begin{array}{l}2 \text { (metformin }+ \\
\text { SGLT2 inhibitors) }\end{array}$} & 60 & $\begin{array}{l}\text { Metformin 1,000 } \\
\text { mg (Glucophage }{ }^{\circledR} \text { ) }\end{array}$ & $\begin{array}{l}\text { Dapagliflozin/metformin } 5 \\
\mathrm{mg} / 1,000 \mathrm{mg}\left(\text { Xigduo }^{\circledR}\right)\end{array}$ & One tablet two times per day \\
\hline & & & $\begin{array}{l}\text { Empagliflozin/metformin } 5 \\
\mathrm{mg} / 1,000 \mathrm{mg}\left(\text { Synjardy }{ }^{\circledR}\right)\end{array}$ & One tablet two times per day \\
\hline & & & $\begin{array}{l}\text { Empagliflozin/metformin } 12.5 \\
\mathrm{mg} / 1,000 \mathrm{mg}\left(\text { Synjardy }^{\circledR}\right)\end{array}$ & One tablet two times per day \\
\hline
\end{tabular}

DPP4: dipeptidyl peptidase 4; SGLT2: sodium-glucose cotransporter 2

within the total study subjects, groups 1 and 2 respectively. There were significant differences in mean systolic blood pressure $(\mathrm{P}<0.001)$ and diastolic blood pressure $(\mathrm{P}<0.05)$ after add-on therapy within the total study subjects, groups 1 and 2. The mean WC was significantly differed between the two study groups $(\mathrm{P}<0.05)$, but there was no significant difference in the mean weight, BMI, systolic and diastolic blood pressures between the two study groups $(\mathrm{P}>0.05)$.

The mean FPG level in the total study subjects with addon therapy was significantly reduced by -1.8 (95\% CI: -2.0 , -1.6) $\mathrm{mmol} / \mathrm{L}$ from the 12 th week $(\mathrm{P}<0.001)$ compared to a decline with metformin monotherapy which is $-0.45(95 \%$ CI: $-0.66,-0.25) \mathrm{mmol} / \mathrm{L}$ from the baseline $(\mathrm{P}<0.001)$. The mean FPG level in group 1 with add-on therapy was reduced by -1.47 (95\% CI: $-1.64,-1.30) \mathrm{mmol} / \mathrm{L}$ from the 12 th week $(\mathrm{P}$ $<0.001)$. However, there was no significant alteration within group 1 with metformin monotherapy from the baseline $(\mathrm{P}=$ 0.217 ). The mean FPG level in group 2 with add-on therapy was reduced by $-2.14(95 \% \mathrm{CI}:-2.38,-1.91) \mathrm{mmol} / \mathrm{L}$ from the 12th week ( $\mathrm{P}<0.001)$ compared to a decline with metformin monotherapy which is $-0.60(95 \% \mathrm{CI}:-0.80,-0.40) \mathrm{mmol} / \mathrm{L}$ from the baseline $(\mathrm{P}<0.001)$. The mean FPG level significantly differed between the two study groups $(\mathrm{P}=0.02)$.

The mean PPBG in the total study subjects was significantly reduced by $-2.36(95 \% \mathrm{CI}:-2.89,-1.83) \mathrm{mmol} / \mathrm{L}$ from the 12th week $(\mathrm{P}<0.001)$ compared to a decline with metformin monotherapy which is -0.48 (95\% CI: $-0.91,-0.05) \mathrm{mmol} / \mathrm{L}$ from the baseline $(\mathrm{P}<0.001)$. The mean $\mathrm{PPBG}$ level in group 1 was reduced by $-2.36(95 \% \mathrm{CI}:-2.89,-1.83) \mathrm{mmol} / \mathrm{L}$ from the 12 th week $(\mathrm{P}<0.001)$ compared to a decrease with metformin monotherapy which is $-0.48(95 \% \mathrm{CI}:-0.91,-0.05) \mathrm{mmol} / \mathrm{L}$ from the baseline $(\mathrm{P}=0.026)$. The mean $\mathrm{PPBG}$ level in group 2 was reduced by $-3.13(95 \% \mathrm{CI}:-3.63,-2.63) \mathrm{mmol} / \mathrm{L}$ from the 12th week $(\mathrm{P}<0.001)$ compared to a decline with metformin monotherapy which is $-0.62(95 \% \mathrm{CI}:-0.89,-0.35) \mathrm{mmol} / \mathrm{L}$ from the baseline $(\mathrm{P}<0.001)$. The mean $\mathrm{PPBG}$ level significantly altered between the two study groups $(\mathrm{P}=0.001)$.

Add-on therapy significantly reduced the HbAlc levels by $-1.07 \%$ (95\% CI: -1.15, -1.00), -0.96\% (95\% CI: -1.04, -0.89), and $-1.19 \%(95 \% \mathrm{CI}:-1.28,-1.09)$ when compared to metformin monotherapy $(\mathrm{P}<0.001)$ which altered significantly from baseline with a change of $-0.10 \%(95 \% \mathrm{CI}:-0.14,-0.06)$, $-0.08 \%$ (95\% CI: $-0.14,-0.02$ ), and $-0.12 \%$ (95\% CI: -0.17 , $-0.07)$ within the total study subjects, groups 1 and 2 , respectively $(\mathrm{P}<0.001)$. The mean HbA1c significantly differed between the two study groups $(\mathrm{P}=0.008)$. Supplementary Material 1 (wwww.jocmr.org) also shows the change in the lipid profile with a significant difference in mean TC $(\mathrm{P}<0.001)$, TG $(\mathrm{P}<0.001)$, HDL-C $(\mathrm{P}<0.001)$, and LDL-C $(\mathrm{P}<0.001)$ after add-on therapy within the total study subjects, groups 1 and 2. The mean TG and HDL-C level significantly differed between the two study groups $(\mathrm{P}<0.05)$, but there was no significant difference in the mean TC and LDL-C level between the two study groups $(\mathrm{P}>0.05)$. The mean creatinine level was significant between the two study groups $(\mathrm{P}=0.041)$.

The mean RBP4 in the total study subjects was significantly reduced by $-5.57(95 \% \mathrm{CI}:-6.20,-5.04) \mu \mathrm{g} / \mathrm{mL}$ from the 12th week $(\mathrm{P}<0.001)$. The mean RBP4 level in group 1 was decreased by $-5.13(95 \% \mathrm{CI}:-5.94,-4.33) \mu \mathrm{g} / \mathrm{mL}$ from the 12th week $(\mathrm{P}<0.001)$. The mean RBP4 level in group 2 was reduced by $-6.00(95 \% \mathrm{CI}:-6.65,-5.35) \mu \mathrm{g} / \mathrm{mL}$ from the 12 th week $(\mathrm{P}<0.001)$. There was no significant change with metformin monotherapy from the baseline $(\mathrm{P}>0.05)$ within the total study subjects, groups 1 and 2. The mean RBP4 level was not significant between the two study groups $(\mathrm{P}=0.138)$.

The mean FABP4 in the total study subjects was significantly decreased by -2.67 (95\% CI: $-2.97,-2.37) \mathrm{ng} / \mathrm{mL}$ from the 12th week $(\mathrm{P}<0.001)$. The mean FABP4 level in group 1 was reduced by $-2.10(95 \% \mathrm{CI}:-2.42,-1.79) \mathrm{ng} / \mathrm{mL}$ from the 12th week $(\mathrm{P}<0.001)$. The mean FABP4 level in group 2 was decreased by -3.2 (95\% CI: $-3.63,-2.84) \mathrm{ng} / \mathrm{mL}$ from the 12 th week $(\mathrm{P}<0.001)$. There was no significant variation with metformin monotherapy from the baseline $(\mathrm{P}>0.05)$ within the total study subjects, groups 1 and 2. However, the mean FABP4 level was significant between the two study groups ( $\mathrm{P}$ $=0.038$ ).

The mean visfatin level in the total study subjects significantly altered by $-1.54(95 \% \mathrm{CI}:-1.68,-1.41) \mathrm{ng} / \mathrm{mL}$ from 
the 12th week $(\mathrm{P}<0.001)$ compared to a significant increase with metformin monotherapy which is $0.05(0.00,0.10) \mathrm{ng} /$ $\mathrm{mL}$ from the baseline $(\mathrm{P}=0.047)$. The mean visfatin level in group 1 was decreased by $-1.39(95 \% \mathrm{CI}:-1.58,-1.20) \mathrm{ng} /$ $\mathrm{mL}$ from the 12 th week $(\mathrm{P}<0.001)$. There was no significant variation with metformin monotherapy from the baseline $(\mathrm{P}$ $>0.05)$ within group 1 . The mean visfatin level within group 2 was decreased by -1.69 (95\% CI: $-1.86,-1.53) \mathrm{ng} / \mathrm{mL}$ from the 12 th week $(\mathrm{P}<0.001)$ compared to a significant increase with metformin monotherapy which is $0.07(0.02,0.12) \mathrm{ng} / \mathrm{mL}$ from the baseline $(\mathrm{P}=0.002)$. The mean visfatin level was not significant between the two study groups $(\mathrm{P}=0.265)$.

The mean adiponectin in the total study subjects significantly altered by 3.89 (95\% CI: $3.67,4.12) \mu \mathrm{g} / \mathrm{mL}$ from the 12 th week $(\mathrm{P}<0.001)$. The mean adiponectin level in group 1 was increased by $3.15(95 \%$ CI: $2.89,3.42) \mu \mathrm{g} / \mathrm{mL}$ from the 12th week $(\mathrm{P}<0.001)$. The mean adiponectin level in group 2 was enhanced by 4.63 (95\% CI: 4.33, 4.93) $\mu \mathrm{g} / \mathrm{mL}$ from the 12th week $(\mathrm{P}<0.001)$. There was no significant difference with metformin monotherapy from the baseline $(\mathrm{P}>$ 0.05 ) within total study participants, groups 1 and 2 . The mean adiponectin level was not significant between the two study groups $(\mathrm{P}=0.736)$.

Treatment-emergent adverse events during the study were the incidence of hypoglycemia $(n=1)$ with add-on therapy of DPP4 inhibitors, urinary tract infections $(n=2)$ and genital infections $(n=1)$ with add-on therapy of SGLT2 inhibitors.

\section{Discussion}

Metformin is the most preferred first-line therapy recommended by most guideline committees for type 2 diabetes mellitus despite changes in lifestyle and dietary habits $[23,24]$. The recent guidelines on the treatment of type 2 diabetes recommend first and second-line therapies to prevent the patient's cardiovascular risk profile $[25,26]$. The guidelines also suggest that SGLT2 inhibitors are more suitable than DPP4 inhibitors or sulfonylureas as add-on therapy to metformin [25, 27]. The recommendations are given based on the trials that included type 2 diabetic patients with cardiovascular disease [25].

The current study is the first investigation on the effects of add-on therapy of SGLT2 inhibitors and DPP4 inhibitors on visceral fat-associated adipokines. The study subjects had an inactive lifestyle with a regular controlled diet during the study period. The study findings suggest that adipokine levels are altered extensively after the add-on therapy in type 2 diabetic patients inadequately controlled with metformin. The result indicates a substantial effect $(\mathrm{P}<0.001)$ of the add-on therapy on adipokines in type 2 diabetic patients. The result also indicates a significant effect $(\mathrm{P}<0.05)$ of the add-on therapy on clinical parameters such as weight, BMI, WC, systolic and diastolic blood pressures in type 2 diabetic patients. The study provides new insight into the effective therapeutic target in central obese type 2 diabetic patients.

Adipokines regulate insulin sensitivity, endothelial function, energy expenditure, glucose and lipid metabolism and inflammation [28]. Abdominal fat deposition induces several metabolic variations that result in increased hepatic glucose output and decreased insulin sensitivity in adipose tissue, leading to diabetes mellitus [29]. An increase in adiponectin level reduces obesity-related diseases, insulin resistance, type 2 diabetes and cardiovascular disease [30]. Adiponectin has insulin-sensitizing, anti-inflammatory and cardiovascular modulating effects in type 2 diabetic patients. Adiponectin can provide an important therapeutic tool to reduce the burden associated with obesity, diabetes and cardiovascular disease [31]. Our study reveals that adiponectin level is significantly elevated $(\mathrm{P}<0.001)$ with add-on therapy of SGLT2 inhibitors and DPP4 inhibitors.

RBP4 is associated with cardiovascular risk in type 2 diabetic subjects with varying clinical presentations [32]. Recent studies demonstrated that RBP4 levels significantly alter with variables related to insulin resistance, obesity, type 2 diabetes and metabolic syndrome [32-34]. Furthermore, there is some indication that reduced RBP4 levels decrease the risk of diabetic nephropathy [34]. The plasma level of RBP4 is higher in type 2 diabetic patients when compared with normal controls [35]. Our study shows that serum RBP4 is significantly reduced $(\mathrm{P}<0.001)$ with add-on therapy of SGLT2 inhibitors and DPP4 inhibitors.

Recent studies link FABP4 with insulin resistance and type 2 diabetes mellitus $[35,36]$. FABP4 plays a vital role in regulating lipid metabolism and inflammation [35, 37]. Recent research reveals that FABP4 is a predictor for developing atherosclerosis, cardiovascular risk, metabolic syndrome and type 2 diabetes mellitus $[35,38]$. The circulating FABP4 levels influence the clinical parameters such as BMI, dyslipidemia and insulin resistance. FABP4 appears to be one of the most considerable interests to develop potential therapeutic strategies targeting the pathogenesis of metabolic disorders [35]. Treatment with anagliptin decreases the FABP4 concentration in type 2 diabetic patients who are at high risk for cardiovascular disease receiving statin therapy [39]. Our study shows that FABP4 level is significantly decreased $(\mathrm{P}<0.001)$ with add-on therapy of SGLT2 inhibitors and DPP4 inhibitors. The study also demonstrates that SGLT2 inhibitors are more effective $(\mathrm{P}<0.05)$ on serum FABP4 in patients with type 2 diabetes.

Visfatin plays a vital role in regulating various pathophysiological functions and is a marker of inflammation and endothelial dysfunction [40]. The visfatin level is linked with vascular inflammation, atherosclerosis and cardiovascular disease in patients with type 2 diabetes [40, 41]. However, the mechanism by which the visfatin levels influences atherosclerosis plaque progression in patients with type 2 diabetes remains unclear [40]. Our study explains that visfatin level is significantly decreased with add-on therapy of SGLT2 inhibitors and DPP4 inhibitors.

SGLT2 inhibitors significantly decrease FPG and HbA1c in type 2 diabetic patients in clinical practice. The patients treated with SGLT2 inhibitors showed a decline in HbA1c of about $1 \%$ after 18 weeks of the study period. A part of the participants achieved the glycemic targets set by ADA, thus strictly relating the effectiveness of SGLT2 inhibitors in randomized clinical trials [31]. Previous studies reveal that FPG and $\mathrm{HbAlc}$ levels showed more significant reductions with dapagliflozin treatment than with placebo. The therapy with 
SGLT2 inhibitor might be more suitable in type 2 diabetic patients with visceral obesity and poor glycemic control. SGLT2 inhibitor also has a positive influence on glycemic control without the appearance of hypoglycemia [42]. Our study found that the add-on therapy of both SGLT2 inhibitors and DPP4 inhibitors is associated with a significant beneficial effect $(\mathrm{P}$ $<0.001$ ) on FPG, PPBG and HbA1c levels in type 2 diabetic patients. The study also demonstrates that SGLT2 inhibitors are more effective $(\mathrm{P}<0.05)$ on FPG, PPBG and HbAlc in patients with type 2 diabetes.

The different effects on plasma lipid levels have been reported in some studies. SGLT2 inhibitors target renal glucose reabsorption via an insulin-independent mechanism, resulting in the loss of glucose. Recent evidence supports that using SGLT2 inhibitors in regular treatment in type 2 diabetic patients with high cardiovascular risk can have beneficial effects in clinically relevant cardiovascular outcomes [43, 44]. SGLT2 inhibitors are more effective in improving HDL-C from baseline to 24 weeks than DPP4 inhibitor therapy, suggesting that dapagliflozin is the preferred choice in type 2 diabetic patients with low HDL-C [44]. The mechanism by which the DPP4 inhibitor influences lipid metabolism in patients with type 2 diabetes is not fully understood. The effect may be due to glucagon-like peptide-1 receptor-mediated inhibitory impact on lipid intake in the gastrointestinal tract $[44,45]$. Our study found that the add-on therapy has a significant beneficial effect $(\mathrm{P}<0.001)$ on lipid profile in type 2 diabetic patients. The study also demonstrates that SGLT2 inhibitors are more effective $(\mathrm{P}<0.05)$ on TC, TG, VLDL and HDL-C in patients with type 2 diabetes.

There were few limitations to this study. The sample size was relatively small due to the strict restriction of participants who have taken only metformin therapy. The study also excluded the patients who have taken the statins during the follow-up period. The participants received varying doses of DPP4 inhibitors and SGLT2 inhibitors. However, the dosage was the same in each medication given. The study excluded the patients who changed the drug during the follow-up periods. Also, the participants' lifestyle was different as the study included different nationalities having various dietary habits. The participants who developed adverse clinical events during the treatment period were discontinued from the study.

\section{Conclusion}

The adipokine levels are altered substantially with the addon therapy in type 2 diabetic patients inadequately controlled with metformin. Add-on therapy of both SGLT2 inhibitors and DPP4 inhibitors substantially affects RBP4, FABP4, adiponectin and visfatin in type 2 diabetic patients. However, the SGLT2 inhibitors are more effective on serum FABP4 in patients with type 2 diabetes. The add-on therapy exerts a beneficial effect by altering the visceral fat-associated adipokine levels and controlling metabolic activities. The SGLT2 inhibitors and DPP4 inhibitors also reduce cardiovascular risk in patients with type 2 diabetes. The study provides new insight into the effective therapeutic goal in central obese type 2 diabetic patients.

\section{Supplementary Material}

Suppl 1. Effect of Add-On Therapy of DPP4 Inhibitors and SGLT2 Inhibitors on Clinical Parameters, Biochemical Variables and Adipokines.

\section{Acknowledgments}

We acknowledge the laboratory staff and biomedical engineer in Thumbay Laboratory, Gulf Medical University for their help and support during the research work.

\section{Financial Disclosure}

There was no specific funding source to be mentioned.

\section{Conflict of Interest}

The authors report no conflict of interest.

\section{Informed Consent}

All subjects provided written informed consent.

\section{Author Contributions}

Abid Shaheer performed the study and drafted the manuscript. Ashok Kumar has supervised the study, manuscript writing and editing. Palat Menon also guided the study and provided facility support for the research. Mahir Jallo and Shaikh Basha are the clinicians who assisted in the selection of patients in the study.

\section{Data Availability}

The authors declare that data supporting the findings of this study are available within the article.

\section{References}

1. Khemka VK. Metabolic risk factors in obesity and diabetes mellitus: implications in the pathogenesis and therapy. Integr Obesity Diabetes. 2017;3(3):1-4.

2. Bosomworth NJ. Normal-weight central obesity: Unique hazard of the toxic waist. Can Fam Physician. 2019;65(6):399-408.

3. Valeska O, Soumyalekshmi N, Omar E, Claudio A, Carlos S, Felipe AZ. Association between insulin resistance and the development of cardiovascular disease. Cardiovasc Diabetol. 2018;17(1):122. 
4. Cercato C, Fonseca FA. Cardiovascular risk and obesity. Diabetol Metab Syndr. 2019;11(74):1-15.

5. Neeland IJ, Poirier P, Despres JP. Cardiovascular and metabolic heterogeneity of obesity: clinical challenges and implications for management. Circulation. 2018;137(13):13911406.

6. Shibata R, Ouchi N, Ohashi K, Murohara T. The role of adipokines in cardiovascular disease. J Cardiol. 2017; 70(4):329-334.

7. Karuranga S, Malanda B, Saeedi P, Salpea P. The IDF Diabetes Atlas, 9th edition. Int Diab Feder. 2019:62-77.

8. Sulaiman N, Elbadawi S, Hussein A, Abusnana S, Madani A, Mairghani M, Alawadi F, et al. Prevalence of overweight and obesity in United Arab Emirates Expatriates: the UAE National Diabetes and Lifestyle Study. Diabetol Metab Syndr. 2017;9:88.

9. Rena G, Hardie DG, Pearson ER. The mechanisms of action of metformin. Diabetologia. 2017;60(9):1577-1585.

10. LaMoia TE, Shulman GI. Cellular and molecular mechanisms of metformin action. Endocr Rev. 2021;42(1):77-96.

11. Horakova O, Kroupova P, Bardova K, Buresova J, Janovska P, Kopecky J, Rossmeisl M. Metformin acutely lowers blood glucose levels by inhibition of intestinal glucose transport. Sci Rep. 2019;9(1):6156.

12. Hsia DS, Grove O, Cefalu WT. An update on SGLT2 inhibitors for the treatment of diabetes mellitus. Curr Opin Endocrinol Diabetes Obes. 2017;24(1):73-79.

13. Simes BC, MacGregor GG. Sodium-Glucose Cotransporter-2 (SGLT2) inhibitors: a clinician's guide. Diabetes Metab Syndr Obes. 2019;12:2125-2136.

14. Kurinami N, Sugiyama S, Nishimura H, Morita A, Yoshida A, Hieshima K, Miyamoto F, et al. Clinical factors associated with initial decrease in body-fat percentage induced by add-on sodium-glucose co-transporter 2 inhibitors in patient with type 2 diabetes mellitus. Clin Drug Investig. 2018;38(1):19-27.

15. Diaz-Rodriguez E, Agra RM, Fernandez AL, Adrio B, Garcia-Caballero T, Gonzalez-Juanatey JR, Eiras S. Effects of dapagliflozin on human epicardial adipose tissue: modulation of insulin resistance, inflammatory chemokine production, and differentiation ability. Cardiovasc Res. 2018;114(2):336-346.

16. Muller-Wieland D, Kellerer M, Cypryk K, Skripova D, Rohwedder K, Johnsson E, Garcia-Sanchez R, et al. Efficacy and safety of dapagliflozin or dapagliflozin plus saxagliptin versus glimepiride as add-on to metformin in patients with type 2 diabetes. Diabetes Obes Metab. 2018;20(11):2598-2607.

17. Softeland E, Meier JJ, Vangen B, Toorawa R, Maldonado-Lutomirsky M, Broedl UC. Empagliflozin as add-on therapy in patients with type 2 diabetes inadequately controlled with linagliptin and metformin: a 24-week randomized, double-blind, parallel-group Trial. Diabetes Care. 2017;40(2):201-209.

18. Silva Junior WS, Souza M, Nogueira Neto JF, Bouskela E, Kraemer-Aguiar LG. Dipeptidyl peptidase 4 activity is related to body composition, measures of adiposity, and insulin resistance in subjects with excessive adiposity and different degrees of glucose tolerance. J Diabetes Res.
2019;2019:5238013.

19. Wang X, Zheng P, Huang G, Yang L, Zhou Z. Dipeptidyl peptidase-4(DPP-4) inhibitors: promising new agents for autoimmune diabetes. Clin Exp Med. 2018;18(4):473480 .

20. Shi C, Zhang R, Bai R, Liu D, Wang Y, Zhang X, Wang $\mathrm{H}$, et al. Efficacy and safety of sitagliptin added to metformin and insulin compared with voglibose in patients with newly diagnosed type 2 diabetes. Clinics (Sao Paulo). 2019;74:e736.

21. Hayashi T, Murayama H, Shinfuku Y, Taniguchi T, Tsumiyama I, Oyama N. Safety and efficacy of vildagliptin: 52-week post-marketing surveillance of Japanese patients with type 2 diabetes in combination with other oral antidiabetics and insulin. Expert Opin Pharmacother. 2020;21(1):121-130.

22. Zhou XJ, Ding L, Liu JX, Su LQ, Dong JJ, Liao L. Efficacy and short-term side effects of sitagliptin, vildagliptin and saxagliptin in Chinese diabetes: a randomized clinical trial. Endocr Connect. 2019;8(4):318-325.

23. Sanchez-Rangel E, Inzucchi SE. Metformin: clinical use in type 2 diabetes. Diabetologia. 2017;60(9):1586-1593.

24. Chelsea B, Cimmaron RS, Vatsala S, Renee P, Victoria A, Neda R. Should metformin remain the first-line therapy for treatment of type 2 diabetes. Ther Adv Endocrinol Metab. 2021;12:2042018820980225.

25. Thein D, Christiansen MN, Mogensen UM, Bundgaard JS, Rorth R, Madelaire C, Fosbol EL, et al. Add-on therapy in metformin-treated patients with type 2 diabetes at moderate cardiovascular risk: a nationwide study. Cardiovasc Diabetol. 2020;19(1):107.

26. Mosenzon O, Pollack R, Raz I. Treatment of type 2 diabetes: from "Guidelines" to "Position Statements" and back: recommendations of the israel national diabetes council. Diabetes Care. 2016;39(Suppl 2):S146-153.

27. Kalra S, Kesavadev J, Chadha M, Kumar GV. Sodium-glucose cotransporter-2 inhibitors in combination with other glucose-lowering agents for the treatment of type 2 diabetes mellitus. Indian J Endocrinol Metab. 2018;22(6):827836.

28. Calcaterra V, Regalbuto C, Porri D, Pelizzo G, Mazzon E, Vinci F, Zuccotti G, et al. Inflammation in obesity-related complications in children: the protective effect of diet and its potential role as a therapeutic agent. Biomolecules. 2020;10(9):1324.

29. Frankenberg ADV, Reis AF, Gerchman F. Relationships between adiponectin levels, the metabolic syndrome, and type 2 diabetes: a literature review. Arch Endocrinol Metab. 2017;61(6):614-622.

30. Achari AE, Jain SK. Adiponectin, a therapeutic target for obesity, diabetes, and endothelial dysfunction. Int J Mol Sci. 2017;18(6):1321.

31. Mirabelli M, Chiefari E, Caroleo P, Vero R, Brunetti FS, Corigliano DM, Arcidiacono B, et al. Long-term effectiveness and safety of SGLT-2 inhibitors in an italian cohort of patients with type 2 diabetes mellitus. J Diabetes Res. 2019;2019:3971060.

32. Li JY, Chen XX, Lu XH, Zhang CB, Shi QP, Feng L. Elevated RBP4 plasma levels were associated with 
diabetic retinopathy in type 2 diabetes. Biosci Rep. 2018;38(5):BSR20181100.

33. Wang Y, Sun L, Lin X, Yuan JM, Koh WP, Pan A. Retinol binding protein 4 and risk of type 2 diabetes in Singapore Chinese men and women: a nested case-control study. Nutr Metab (Lond). 2019;16:3.

34. Zhang L, Cheng YL, Xue S, Xu ZG. The role of circulating RBP4 in the type 2 diabetes patients with kidney diseases: a systematic review and meta-analysis. Dis Markers. 2020;2020:8830471.

35. Trojnar M, Patro-Malysza J, Kimber-Trojnar Z, Leszczynska-Gorzelak B, Mosiewicz J. Associations between fatty acid-binding protein 4(-)A proinflammatory adipokine and insulin resistance, gestational and type 2 diabetes mellitus. Cells. 2019;8(3):227.

36. Nakamura R, Okura T, Fujioka Y, Sumi K, Matsuzawa K, Izawa $\mathrm{S}$, Ueta $\mathrm{E}$, et al. Serum fatty acid-binding protein 4 (FABP4) concentration is associated with insulin resistance in peripheral tissues, A clinical study. PLoS One. 2017;12(6):e0179737.

37. Qiao Y, Liu L, Yin L, Xu L, Tang Z, Qi Y, Mao Z, et al. FABP4 contributes to renal interstitial fibrosis via mediating inflammation and lipid metabolism. Cell Death Dis. 2019;10(6):382.

38. Saito N, Furuhashi M, Koyama M, Higashiura Y, Akasaka H, Tanaka M, Moniwa N, et al. Elevated circulating FABP4 concentration predicts cardiovascular death in a general population: a 12-year prospective study. Sci Rep. 2021;11(1):4008.

39. Furuhashi M, Sakuma I, Morimoto T, Higashiura Y, Sakai
A, Matsumoto M, Sakuma M, et al. Treatment with anagliptin, a DPP-4 inhibitor, decreases FABP4 concentration in patients with type 2 diabetes mellitus at a high risk for cardiovascular disease who are receiving statin therapy. Cardiovasc Diabetol. 2020;19(1):89.

40. Zheng LY, Xu X, Wan RH, Xia S, Lu J, Huang Q. Association between serum visfatin levels and atherosclerotic plaque in patients with type 2 diabetes. Diabetol Metab Syndr. 2019;11:60.

41. Duman H, Ozyildiz AG, Bahceci I, Duman H, Uslu A, Ergul E. Serum visfatin level is associated with complexity of coronary artery disease in patients with stable angina pectoris. Ther Adv Cardiovasc Dis. 2019;13:1753944719880448.

42. Luo M, Kong X, Wang H, Zhai X, Cai T, Ding B, Hu Y, et al. Effect of dapagliflozin on glycemic variability in patients with type 2 diabetes under insulin glargine combined with other oral hypoglycemic drugs. J Diabetes Res. 2020;2020:6666403.

43. Vallon V, Thomson SC. Targeting renal glucose reabsorption to treat hyperglycaemia: the pleiotropic effects of SGLT2 inhibition. Diabetologia. 2017;60(2):215-225.

44. Cha SA, Park YM, Yun JS, Lim TS, Song KH, Yoo KD, Ahn YB, et al. A comparison of effects of DPP-4 inhibitor and SGLT2 inhibitor on lipid profile in patients with type 2 diabetes. Lipids Health Dis. 2017;16(1):58.

45. Konstantinos M. The role of DPP-4 inhibitors in the treatment algorithm of type 2 diabetes mellitus: when to select, what to expect. Int J Environ Res Public Health. 2019;16(15):2720. 